\title{
HOMOTOPY TYPE COMPARISON OF A SPACE WITH COMPLEXES ASSOCIATED WITH ITS OPEN COVERS ${ }^{1}$
}

\author{
MICHAEL C. MCCORD
}

1. Introduction. This paper deals with the homotopy type comparison of a space with certain complexes (such as the nerve) associated with suitably "well pieced together" open covers of the space by homotopically trivial sets. The main theorem (Theorem 1) implies a slightly different version (Theorem 2) of a theorem of A. Weil [7]. The method of proof is quite different from Weil's. A simple use is made of a theorem in [6] of Alexandroff's [1] "discrete spaces" (spaces in which the intersection of any collection of open sets is open). Also use is made of a modification (Theorem 3) of a theorem of A. Dold and R. Thom [3].

It is conceivable that Theorem 1 could contribute to a positive solution of the unsolved problem: Does every compact topological manifold have the homotopy type of a finite complex? See the discussion following the statement of the theorem.

Other results to which Theorem 1 is related were obtained by J. Leray [5] and by K. Borsuk [2].

2. Notation and terminology. Suppose $\mathcal{u}$ is an open cover of a space $X$ (a collection of nonempty open subsets of $X$ whose union is $X$ ). Let $N(\mathcal{u})$ denote the nerve of $\mathcal{u}$. More than with $N(\mathcal{U})$, we shall be concerned with the following subcomplex of $N(\mathcal{u})$. Let $K(\mathcal{u})$ be the complex whose vertices are the members of $u$ and whose simplexes are the finite totally ordered subcollections of $\mathcal{u}$ (where $\mathcal{u}$ is partially ordered by inclusion). In general $K(\mathcal{U})$ does not have the same homotopy type as $N(\mathcal{u})$.

The open cover $\mathcal{U}$ will be called basis-like if the intersection of any two members of $u$ is a union of members of $u$. This is equivalent to saying that $\mathcal{U}$ is a basis for a topology on $X$ smaller than the given one. An open cover $\mathcal{U}$ is point-finite if each point of $X$ is contained in only finitely many members of $\mathcal{u}$.

A map $f: X \rightarrow Y$ is a weak homotopy equivalence if the induced maps $f_{*}: \pi_{i}(X, x) \rightarrow \pi_{i}(Y, f x)$ are isomorphisms for all $x \in X$ and all $i \geqq 0$. A space $X$ is homotopically trivial if $\pi_{i}(X, x)=0$ for all $i \geqq 0$.

If $K$ is an (abstract) simplicial complex, $|K|$ denotes the underlying polyhedron with the weak topology.

Received by the editors May 31, 1966.

1 This research was supported in part by NSF Grant GP 6016. 


\section{Statement of results.}

Theorem 1. Let $X$ be a space and let $u$ be a point-finite, basis-like, open cover of $X$ by homotopically trivial sets. Then there exists a weak homotopy equivalence $f:|K(\mathcal{u})| \rightarrow X$.

REMARK. Of course if in addition we know that $X$ has the homotopy type of a CW-complex (for example when $X$ is a topological manifold), then we may conclude from a theorem of J. H. C. Whitehead [8] that $f$ is an actual homotopy equivalence.

Thus the problem mentioned in $\$ 1$ has a positive solution if the following question has an affirmative answer: Does every compact topological manifold possess a finite, basis-like, open cover by contractible sets? (In this context "contractible" is equivalent to "homotopically trivial.") Any counterexample to this would be a counterexample to the triangulation problem, because every finite polyhedron has such an open cover: the open stars of its simplexes. However, perhaps this question could be answered affirmatively without solving the triangulation problem.

In $\S 5$, Theorem 1 will be used to derive the following variation on a theorem of Weil.

Theorem 2 (cF. Weil [7, p. 141]). Let $X$ be a space and let $v$ be a point-finite open cover of $X$ such that the intersection of any (finite) subcollection of $\mathcal{V}$ is homotopically trivial. Then there exists a weak homotopy equivalence $|N(\mathrm{~V})| \rightarrow X$.

In Weil's version, it is assumed that $X \times X \times[0,1]$ is normal, that $V$ is locally finite, and that the intersections of subcollections of $V$ are solid; and it is concluded that there is an actual homotopy equivalence $|N(\mathcal{V})| \rightarrow X$. If we replace the first of these assumptions by the assumption that $X$ is separable metric, then we can derive Weil's conclusion, because then by a theorem of O. Hanner [4, p. 392], $X$ is an ANR, so that Whitehead's theorem applies.

There are simple examples to which Theorem 1 applies, but where $|N(\mathcal{U})|$ does not have the (weak) homotopy type of $X$.

The main tool in proving Theorem 1 is the following theorem used in [6]. This theorem follows from a modification of the proof of Satz 2.2 of Dold and Thom [3]. For details on the necessary modification, see [6].

Theorem 3. Suppose $p$ is a map of a space $X$ into a space $Y$ and suppose there exists a basis-like open cover w of $Y$ satisfying the following condition: For each $W \in W$, the restriction $p \mid p^{-1}(W): p^{-1}(W)$ $\rightarrow W$ is a weak homotopy equivalence. Then $p$ itself is a weak homotopy equivalence. 
4. Proof of Theorem 1. First let us state the following

LemMa 1. If in the diagram

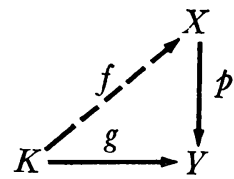

$K$ is a CW-complex and $p$ is a weak homotopy equivalence, then $f$ can be found making the diagram homotopy commutative. Hence if $g$ is also a weak homotopy equivalence, then so is $f$.

The proof, which is simple and involves the mapping cylinder of $p$, appears as a part of an argument on page 244 of [3].

Now suppose $X$ and $\mathcal{U}$ are as in the statement of Theorem 1. Using the fact that $u$ is partially ordered by inclusion and using the modification in [6] of Alexandroff's [1] procedure, we make $\mathcal{u}$ in to a topological space as follows. For each $U \in \mathcal{u}$, let $[U]=\{V \in \mathcal{~}: V \subset U\}$. Then as a basis for the required topology we take the collection $\{[U]: U \in u\}$.

As in [6], we define a map $g:|K(\mathcal{u})| \rightarrow \mathcal{u}$ as follows: If $x \in|K(\mathcal{u})|$, then let $\left(U_{0}, \cdots, U_{n}\right)$ be the unique open simplex of $|K(\mathcal{u})|$ to which $x$ belongs, with $U_{0} \subset \cdots \subset U_{n}$. Then $g(x)=U_{0}$. In [6] it is shown that $g$ is continuous and, by use of Theorem 3 , that $g$ is a weak homotopy equivalence.

Next we define a map $p: X \rightarrow u$. For each $x \in X$ let $p(x)$ be the smallest member of $u$ containing $x$. This exists since $u$ is point-finite and basis-like. The proof of the following lemma is straightforward.

Lemma 2. For each $U \in \mathcal{U}, p^{-1}([U])=U$.

Since the sets $[U]$ form (by definition) a basis for the space $\mathcal{u}$, this implies that $p$ is continuous.

Now we wish to apply Theorem 3 to show that $p$ is a weak homotopy equivalence. For the basis-like open cover of $u$ we take simply the basis $W=\{[U]: U \in \mathcal{U}\}$. It is shown in [6] that each $[U]$ is a contractible subset of $\mathcal{u}$. By Lemma $2, p^{-1}([U])=U$; and $p \mid U: U \rightarrow[U]$ is trivially a weak homotopy equivalence, since $U$ is assumed to be homotopically trivial. Hence Theorem 3 implies that $p$ is a weak homotopy equivalence. Now we apply Lemma 1 to obtain a weak homotopy equivalence $f:|K(\mathcal{U})| \rightarrow X$.

5. Proof of Theorem 2. Let $u$ be the collection of all nonempty intersections of (finite) subcollections of $\mathcal{v}$. Clearly $u$ satisfies the conditions of Theorem 1, so that we get a weak homotopy equiva- 
lence $|K(\mathcal{u})| \rightarrow X$. From the facts that $v \subset \mathcal{C}$ and that $u$ is a refinement of $V$, it is easy to see that $|N(\mathcal{V})|$ is a deformation retract of $|N(\mathcal{U})|$. (More generally, if two covers of a space refine each other, then their nerves have the same homotopy type.) Hence the proof of the theorem is completed by the following

Lemma 3. Let $u$ be a cover of a space with the property that the intersection of any finite subcollection of $u$ is either empty or a member of $u$. Then $|K(\mathcal{U})|$ is a deformation retract of $|N(\mathcal{U})|$.

Proof. Abbreviate $K=K(\mathcal{U}), N=N(\mathcal{U})$, and let $N^{\prime}$ be the first barycentric subdivision of $N$. Let us define a simplicial map $\phi: N^{\prime} \rightarrow K$ as follows. The vertices of $N^{\prime}$ are the barycenters $b(\sigma)$ of the simplexes $\sigma$ of $N$. Define $\phi$ on these vertices by the equation

$$
\phi(b(\sigma))=\text { Carrier } \sigma
$$

(By assumption Carrier $\sigma$, the intersection of the vertices of $\sigma$, being nonempty, is a member of $\mathcal{u}$, that is, a vertex of $K$.) Any simplex of $N^{\prime}$ is spanned by vertices $b\left(\sigma_{0}\right), \cdots, b\left(\sigma_{n}\right)$, where $\sigma_{0} \subset \cdots \subset \sigma_{n}$. Then Carrier $\sigma_{0} \supset \ldots \supset$ Carrier $\sigma_{n}$, so that these vertices span a simplex of $K$. Thus $\phi$ is simplicial.

Let us show that $\phi$, as a map of $|N|=\left|N^{\prime}\right|$ in to $|K|$, is a deformation retraction. Clearly it is a retraction. It suffices then to show that for each simplex $\tau$ of $N^{\prime}$, both $\tau$ and $\phi(\tau)$ are subsets of some simplex $\sigma$ of $N$. Let the vertices of $\tau$ be $b\left(\sigma_{0}\right), \cdots, b\left(\sigma_{n}\right)$, where $\sigma_{0} \subset \cdots \subset \sigma_{n}$, and let the vertices of $\sigma_{n}$ be $U_{0}, \cdots, U_{r}$. We can take $\sigma$ to be the simplex of $N$ spanned by the vertices

$$
\left\{U_{0}, \cdots, U_{r}, \text { Carrier } \sigma_{0}, \cdots, \text { Carrier } \sigma_{n}\right\} .
$$

1. P. S. Alexandroff, Diskrete Räume, Mat. Sb. 2 (1937), 501-518.

2. K. Borsuk, On the imbedding of systems of compacta in simplicial complexes, Fund. Math. 35 (1948), 217-234.

3. A. Dold and R. Thom, Quasifaserungen und unendliche symmetrische Produkte, Ann. of Math. (2) 67 (1958), 239-281.

4. O. Hanner, Some theorems on absolute neighborhood retracts, Ark. Mat. 1 (1950), 389-408.

5. J. Leray, L'anneau spectral et l'anneau filtrê d'homologie d'un espace localement compact et d'une application continue, J. Math. Pures Appl. (9) 29 (1950), 1-139.

6. M. C. McCord, Singular homology groups and homotopy groups of finite topological spaces, Duke Math. J. 33 (1966), 465-474.

7. A. Weil, Sur les théorèmes de de Rham, Comment. Math. Helv. 26 (1952), 119145.

8. J. H. C. Whitehead, Combinatorial homotopy. I, Bull. Amer. Math. Soc. 55 (1949), 213-245. 